\title{
Hepatectomía en 2 tiempos con técnica de ALPPS (Asociación de bipartición hepática con ligadura portal para hepatectomía en 2 tiempos). Experiencia y resultados iniciales del Hospital Regional de Talca
}

\author{
César Muñoz C.., 2 , Guillermo Sepúlveda D. ${ }^{1,2}$, Álvaro Tapia V. ${ }^{1,2}$, Gloria Vergara A. ${ }^{2}$, \\ Marcelo Santelices B. ${ }^{2}$, Rodrigo Vega F. ${ }^{3}$ y Hernán Araya M. ${ }^{4,5}$
}

2 stage hepatectomy with ALPPS technique (Liver splitting association) with ligature portal for hepatectomy in 2 times). Experience and initial results of the Regional Hospital of Talca

Introduction: Postoperative liver failure (POLF) is a relevant cause of morbimortality after liver surgery. An insufficient future liver remnant (FLR) is one of the main causes of POLF. The association of hepatic bipartition with portal ligation for 2-stage hepatectomy (ALPPS) is a relatively new technique that induces rapid parenchymal hypertrophy reducing the waiting time to achieve adequate FLR. Our aim is to communicate the experience and the initial results of the hepatectomy in 2-stages using the ALLPS technique. Material and Method: All patients in whom a 2-stage hepatectomy using ALPPS technique between June 1, 2014 and July 31, 2016 in the Digestive Surgery Unit of the Hospital Regional of Talca were included. Results: There were 47 hepatectomies performed at the Hospital Regional of Talca. In 3 cases $(6.3 \%)$, a 2-stage hepatectomy was performed using the ALPPS technique, in all of which a hypertrophy of the future hepatic remnant (RHF) was achieved, which allowed completing the second stage without POLF clinically significant. Conclusion: In our initial experience, the ALPPS technique successfully allowed increase FLR in patients who initially had insufficient FLR to be submitted a major liver resection.

Key words: hepatectomy; postoperative liver failure; ALPPS.

\section{Resumen}

Introducción: La insuficiencia hepática posoperatoria (IHP) es una causa importante de morbimortalidad posoperatoria en cirugía hepática. Un remanente hepático futuro (RHF) insuficiente es una de las principales causas IHP. La asociación de bipartición hepática con ligadura portal para hepatectomía en 2 tiempos (ALPPS) es una técnica relativamente nueva que induce una rápida hipertrofia parenquimatosa reduciendo el tiempo de espera para lograr un volumen hepático adecuado. Nuestro objetivo es comunicar la experiencia y los resultados iniciales de la hepatectomía en 2 tiempos utilizando la técnica de ALLPS. Material y Método: Se incluyeron todos los pacientes en los que se ha realizado una hepatectomía en 2 tiempos utilizando la técnica de ALPPS entre el 1 de junio de 2014 y el 31 de julio de 2016 en la Unidad de Cirugía Digestiva del Hospital Regional de Talca. Resutados: Durante el periodo de estudio, se realizaron 47 hepatectomías en el Hospital Regional de Talca. En 3 casos (6.3\%), se realizó una hepatectomía en 2 tiempos utilizando la técnica de ALPPS, en todos ellos se logró una hipertrofia del remanente hepático futuro (RHF) que permitió completar el segundo tiempo sin IHP clínicamente significativa. Conclusión: En nuestra experiencia inicial, la técnica de ALPPS logró aumentar el RHF permitiendo resecar pacientes que inicialmente tenían un RHF insuficiente para ser sometidos a una hepatectomía mayor.

Palabras clave: hepatectomía; insuficiencia hepática posoperatoria; ALPPS. 


\section{Introducción}

La cirugía hepática ha avanzado considerablemente en los últimos 20 años ${ }^{1,2}$, otorgando mayor seguridad al procedimiento quirúrgico con reducciones significativas de la morbilidad y mortalidad perioperatoria incluso en hepatectomías mayores ${ }^{3,4}$. Estas mejorías han llevado a expandir las indicaciones a pacientes más complejos y con enfermedad más extensa.

Entre las estrategias para expandir las indicaciones de resecciones hepáticas están: los tratamientos ablativos tumorales con radiofrecuencia o microondas, drogas que actúan directamente disminuyendo la masa tumoral (quimioembolización transarterial) para el tratamiento del hepatocarcinoma o la quimioterapia sistémica en las metástasis hepáticas de cáncer colorrectal.

Una segunda estrategia para aumentar la opción quirúrgica es el manejo de la regeneración hepática y el remanente hepático futuro (RHF) en resecciones extensas múltiples o hepatectomías extendidas para evitar la insuficiencia hepática posoperatoria (IHP). Las principales técnicas para generar un hipertrofia del parénquima hepático son la embolización portal (EP), ligadura portal (LP) y la reciente técnica desarrollada por Hans Schlilt de Regensburg, Alemania, denominada ALPPS por sus iniciales en inglés: Association Liver Partition and Portal vein ligation for Staged hepatectomy, y que consiste en realizar una LP asociado a una transección parenquimatosa del RHF manteniendo el flujo arterial de los 2 fragmentos hepáticos transectados 5 . La principal ventaja teórica de esta técnica por sobre la EP o LP es disminuir el tiempo de espera entre el primer y segundo tiempo en pacientes con una elevada carga tumoral donde la "ventana terapéutica" es reducida.

Nuestro objetivo es comunicar la experiencia y los resultados iniciales de la hepatectomía en 2 tiempos utilizando la técnica de ALLPS en el Hospital Regional de Talca.

\section{Material y Método}

Estudio descriptivo que incluye todos los pacientes sometidos a una hepatectomía en 2 tiempos utilizando la técnica de ALPPS entre el 1 de junio de 2014 y el 31 de julio de 2016 en la Unidad de Cirugía Digestiva del Hospital Regional de Talca.

Todos los pacientes con patología benigna o maligna hepática han sido evaluados por un comité multidisciplinario con gastroenterólogos, radiológos, cirujanos digestivos y oncólogos médicos para establecer la mejor aproximación terapéutica para los pacientes.

En los casos en los cuales se ha planificado una hepatectomía mayor o extendida según la clasificación de Brisbane se realizó una volumetría hepática preoperatoria utilizando el Software Osirix ${ }^{\circledR}$, con una técnica previamente publicada ${ }^{6}$.

Se exponen los 3 primeros casos realizados en nuestro centro, describiendo la indicación, el curso posoperatorio inmediato y la evolución posoperatoria a largo plazo.

\section{Técnica quirúrgica}

Laparotomía de Makucchi o Mercedez-Benz dependiendo de la contextura y la forma del tórax del paciente. Se realizó la sección del ligamento falciforme y los coronarios para poder realizar la ecografía intraoperatoria inicial y ver la relación de las metástasis con las estructuras anatómicas o evidenciar lesiones no diagnosticadas por la resonancia preoperatoria en el lóbulo hepático izquierdo, pero concentrándose principalmente en los segmentos II y III. Pese al poco tiempo de evolución de la técnica se han descrito múltiples variantes del ALPPS. Se realizó la variante clásica o primariamente descrita con transección hepática completa para un RHF de segmento lateral o una fracción del lóbulo hepático izquierdo. Se preparó de rutina la maniobra de Pringle con una cinta, antes de efectuar la transección hepática. Posterior a la resección de las lesiones del lóbulo hepático izquierdo y el segmento lateral, se realizó doble ligadura de la porta derecha con seda $2 / 0$ y se dejó referenciada con una cinta vascular de color azul. Se preservó y referenció la arteria hepática derecha con una cinta vascular de color rojo para facilitar la identificación en el segundo tiempo. La vía biliar derecha se evitó disecar durante esta fase. La transección hepática se realizó con bisturí ultrasónico y pinza bipolar evitando en lo posible la realización de maniobra de Pringle. Una vez completada la transección se aisló el hígado derecho del resto de los órganos abdominales con una bolsa de poliuretano y se instalaron 2 drenajes, 1 en la transección hepática y otro en el espacio retrohepático. El cierre de la laparotomía se efectuó con PDS doble hebra en la aponeurosis y grapas en la piel. Figura 1.

\section{Resultados}

Durante el periodo de estudio, se realizaron 47 hepatectomías en el Hospital Regional de Talca. En 3 casos $(6,3 \%)$, se realizó una hepatectomía en 2 tiempos utilizando la técnica de ALPPS. 


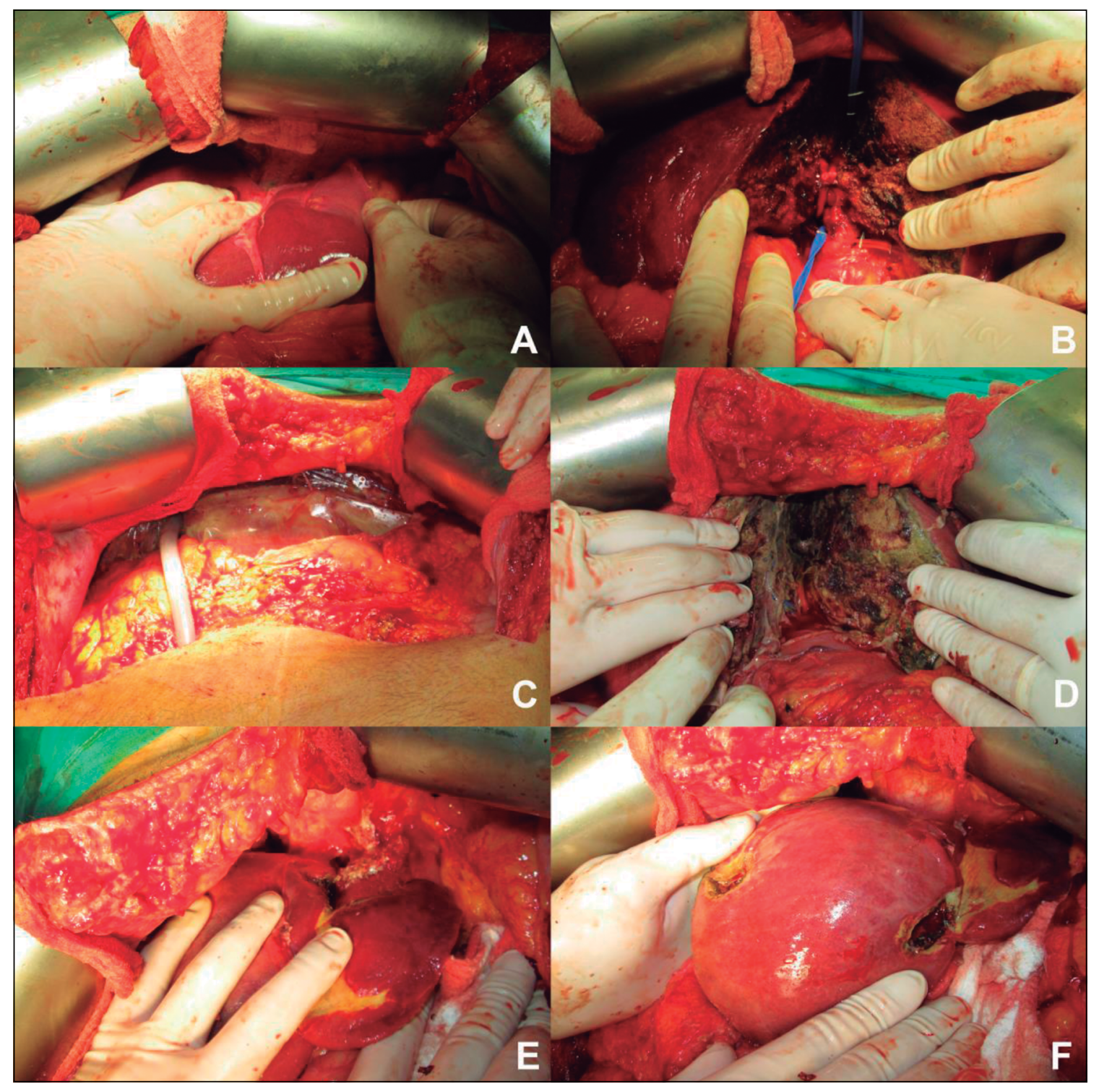

Figura 1. Fotografías que muestran la técnica quirúrgica empleada. A: Segmento lateral pequeño con lesión en el segmento II. B: Transección hepática con cintas vasculares referenciando elementos. C: Visión inicial durante la $2^{\text {a }}$ etapa con el polietileno cubriendo el hígado. D: Superficie de transección posterior a retirar el polietileno. E: Segmento III hipertrofiado y ausencia de segmento II. F: Segmento IV hipertrofia en la $2^{\mathrm{a}}$ etapa.

\section{Caso 1}

Hombre de 61 años, sin antecedentes mórbidos de importancia, inicia estudio por hemorragia digestiva baja. La colonoscopia evidencia lesión proliferativa de colon sigmoides estenosante cuya biopsia informa adenocarcinoma tubular moderadamente diferenciado. El TC de tórax, abdomen y pelvis evidencia 3 lesiones compatibles con metástasis hepáticas en segmentos II, IV y VIII siendo la de mayor tamaño 5,8 cm de diámetro. En su Hospital se decide sigmoidectomía abierta con anastomosis primaria la cual evoluciona satisfactoriamente. Se deriva para evaluación oncológica y se inicia quimioterapia adyuvante con Capecitabina por 9 ciclos con buena tolerancia y respuesta clínica e imagenológica. Se evalúa para resección hepática de las metástasis. Tras la evaluación se planifica hepatectomía derecha y resección del segmento II con una volumetría residual estimada en 335 cc (21\%).

En septiembre de 2015 se realiza laparotomía de Mercedes-Benz y tras la exploración ecográfica se visualiza compromiso del pedículo del segmento II y 2 lesiones de menor tamaño en el segmento IV. Se decide una hepatectomía en 2 tiempos realizando una segmentectomía del II, metastasectomías del IV y transección a nivel de la línea de Cantlie, detalles en Tabla 1. El paciente evoluciona favorablemente y se inició dieta liviana al $2^{\circ}$ de posoperatorio. 
Tabla 1. Resumen de los detalles intraoperatorios de los pacientes

\begin{tabular}{|c|c|c|c|c|c|c|c|c|}
\hline & Etiología & $\mathbf{N}^{0}$ lesiones & Técnica & $\begin{array}{c}\text { T. Op } \\
1^{\text {a }} \text { etapa }\end{array}$ & $\begin{array}{l}\text { Sangrado } \\
1^{\mathrm{a}} \text { etapa }\end{array}$ & Pringle & $\begin{array}{c}\text { T. Op } \\
2^{\mathrm{a}} \text { etapa }\end{array}$ & $\begin{array}{l}\text { Sangrado } \\
2^{\mathbf{a}} \text { etapa }\end{array}$ \\
\hline $\begin{array}{l}\text { Caso } 1 \\
\sigma^{\lambda} 61 \text { años }\end{array}$ & MCCR & 6 & $\mathrm{HD}+\operatorname{seg} \mathrm{II}+\mathrm{NA}$ III-IV & $345^{\prime}$ & $650 \mathrm{cc}$ & $10^{\prime}$ & $150^{\prime}$ & $75 \mathrm{cc}$ \\
\hline $\begin{array}{l}\text { Caso } 2 \\
+45 \text { años }\end{array}$ & MCCR & 7 & $\mathrm{HDE}+\operatorname{seg} \mathrm{III}$ & $257^{\prime}$ & $500 \mathrm{cc}$ & $4^{\prime}$ & $80^{\prime}$ & $100 \mathrm{cc}$ \\
\hline $\begin{array}{l}\text { Caso } 3 \\
+52 \text { años }\end{array}$ & $\mathrm{CCI}$ & 1 & TriD & $283^{\prime}$ & $400 \mathrm{cc}$ & 0 & $85^{\prime}$ & $50 \mathrm{cc}$ \\
\hline
\end{tabular}

MCCR: Metástasis de cáncer colorrectal, CCI: Colangiocarcinoma intrahepático. HD: Hepatectomía derecha, HDE: Hepatectomía derecha extendida, TriD: Trisectorectomía derecha, NA: No anatómica.

Tabla 2. Resumen de las características clínicas de los pacientes

\begin{tabular}{|c|c|c|c|c|c|c|c|c|c|c|}
\hline & QTpre & RHF & $\begin{array}{c}\text { RHF/ } \\
\text { Peso }\end{array}$ & $\begin{array}{c}\text { Tiempo } \\
1^{\circ} \text { a } 2^{\circ}\end{array}$ & HP & Morbilidad & $\begin{array}{l}\text { Estadía } \\
\text { Posop }\end{array}$ & QTpos & Recidiva & Seguimiento \\
\hline $\begin{array}{l}\text { Caso } 1 \\
\delta^{\lambda} 61 \text { años }\end{array}$ & CAPECITABINA & $\begin{array}{c}335 \mathrm{cc} \\
21 \%\end{array}$ & $0,39 \%$ & 7 días & $167 \%$ & No & 21 días & $\begin{array}{c}\text { FOLFIRI } \\
\text { FOLFIRINOX }\end{array}$ & $\begin{array}{l}22 \text { Meses } \\
\text { Hepática }\end{array}$ & $\begin{array}{l}24 \text { Meses } \\
\text { Fallece }\end{array}$ \\
\hline $\begin{array}{l}\text { Caso } 2 \\
q 45 \text { años }\end{array}$ & FOLFOX & $\begin{array}{c}367 \mathrm{cc} \\
24 \%\end{array}$ & $0,43 \%$ & 8 días & $240 \%$ & No & 5 días & FOLFOX & $\begin{array}{c}7 \text { Meses } \\
\text { Extraabdominal }\end{array}$ & $\begin{array}{l}8 \text { Meses } \\
\text { Fallece }\end{array}$ \\
\hline $\begin{array}{l}\text { Caso } 3 \\
+52 \text { años }\end{array}$ & GEM-CIS & $\begin{array}{c}285 \mathrm{cc} \\
22 \%\end{array}$ & $0,41 \%$ & 15 días & $219 \%$ & IIIb & 45 días & GEM-CIS & $\begin{array}{l}12 \text { Meses } \\
\text { Peritoneal }\end{array}$ & $\begin{array}{c}17 \text { Meses } \\
\text { Viva }\end{array}$ \\
\hline
\end{tabular}

QTpre: Quimioterapia preoperatoria, RHF: Remanente hepático futuro, HP: Hipertrofia, QTpos: Quimioterapia posoperatoria.

En la TC de control al $7^{\circ}$ día de posoperatorio se evidencia una volumetría del RHF de $560 \mathrm{cc}$ para los segmentos III y IV con un incremento del $167 \%$, por lo que se decide completar la cirugía. Al $8^{\circ}$ día posoperatorio se completa la hepatectomía derecha sin incidencias intraoperatorias. Evoluciona con ictericia clínica $\left(7,9 \mathrm{mg} / \mathrm{dl}\right.$ al $5^{\circ}$ día), ascitis, hipoprotrombinemia ( $47 \%$ al $5^{\circ}$ día) y sin encefalopatía hepática. Persiste con ictericia que desciende progresivamente. Es dado de alta al $21^{\circ}$ día de posoperatorio.

El paciente completó 10 ciclos de quimioterapia adyuvante con FOLFIRI y luego FOLFIRINOX. En TC de 22 meses de posoperatorio, se detecta recurrencia múltiple en segmento 4 asociado a trombosis de la porta. El paciente fallece a los 24 meses de posoperatorio. El resumen se detalla en Tabla 2.

\section{Caso 2}

Mujer de 45 años, en marzo de 2015 consulta en urgencia por cuadro de dolor abdominal en fosa iliaca derecha. Se realiza TC de tórax, abdomen y pelvis que evidencia tumor de ciego con signos de perforación asociado a metástasis hepáticas múlti- ples. Se realiza colectomía derecha ampliada con íleo transverso anastomosis latero-lateral con técnica de Barcelona. Evoluciona sin complicaciones en el posoperatorio. La biopsia de la pieza quirúrgica evidencia un adenocarcinoma invasor colónico tubular bien diferenciado. Se decide quimioterapia adyuvante con FOLFOX por 10 sesiones con buena tolerancia y respuesta clínica.

La resección $\mathrm{R} 0$ se plantea con una hepatectomía derecha extendida más resección no anatómica del segmento III con un RHF de 367 cc (24,5\%).

Once meses después, se efectúa laparotomía y segmentectomía del III con transección hepática a nivel del segmento IV ligando la vena hepática media (detalles intraoperatorios en Tabla 1). Es extubada a las 24 horas de posoperatorio. En los días posteriores presenta ictericia, ascitis, taquicardia sinusal (130-140x') e hipoprotrombinemia sin encefalopatía. $\mathrm{Al} 7^{\circ}$ día de posoperatorio se realiza TC abdominal con una volumetría de $883 \mathrm{cc}$ lo que equivale a un incremento del $240 \%$. Al $8^{\circ}$ día se completa la $2^{\mathrm{a}}$ etapa, sin incidencias. El posoperatorio es realizado en cuidados intermedios con alta al $5^{\circ}$ día de la $2^{\mathrm{a}}$ etapa. 
La paciente fue evaluada para continuar con quimioterapia adyuvante. Al $6^{\circ}$ mes de posoperatorio presentó un síndrome convulsivo que se estudió con una TC de cerebro evidenciando una lesión metastásica cerebral. La paciente fallece a los 8 meses de posoperatorio. El resumen se detalla en la Tabla 2.

\section{Caso 3}

Mujer de 52 años, en julio de 2015 consulta por cuadro de 2 meses de dolor abdominal y baja de peso. Se realiza TC de abdomen y pelvis que evidencia masa tumoral hepática hipervascular que ocupa gran parte del lóbulo hepático derecho y el segmento IV; con lesiones quísticas atípicas ováricas bilaterales. Se realiza laparotomía exploradora con anexectomía, oforectomía bilateral y biopsia de la lesión hepática. La biopsia de anexos informa lesiones quísticas sin caracteres de malignidad y la lesión hepática es compatible con colangiocarcinoma intrahepático. Se efectúa PET-CT que no informa lesiones hipercaptantes extrahepáticas. Se decide realizar adyuvancia con Gemcitabina y Cisplatino por 3 ciclos. Se completan los 3 ciclos y se realiza nuevo PET-CT que muestra gran masa hepática hipovascular de 14 x $9 \mathrm{~cm}$, hipermetabólica y adenopatías secundarias portocava. El RHF calculado para los segmentos laterales y el caudado de $285 \mathrm{cc}$ $(22 \%)$ Figura $2 \mathrm{~A}$ y $2 \mathrm{C}$.

En julio de 2016 se realiza laparotomía y colecistectomía abierta con biopsia rápida que resulta negativa. Tras realizar la ecografía se decide realiza ALPPS. Los detalles intraoperatorios se describen en la Tabla 1. En el posoperatorio inmediato se traslada a UCI donde se extuba a las 48 horas sin incidencias. Al $6^{\circ}$ día de posoperatorio presenta elevación de parámetros inflamatorios y se inicia tratamiento antibiótico con ceftriaxona y metronidazol. Al $7^{\circ}$ posoperatorio la volumetría es de 624 cc que corresponde a un incremento de $219 \%$ (Figura $2 \mathrm{~B}$ y $2 \mathrm{D}$ ). El $15^{\circ}$ día de posoperatorio se completa la trisectorectomía derecha con hepaticoyeyuno anastomosis a 2 conductos. La paciente evoluciona con ictericia, acolia sin débitos biliares por los drenajes, ante la sospecha de dehiscencia de la hepaticoyeyuno anastomosis se reinterviene, encontrando dehiscencia completa. Se efectúa nueva

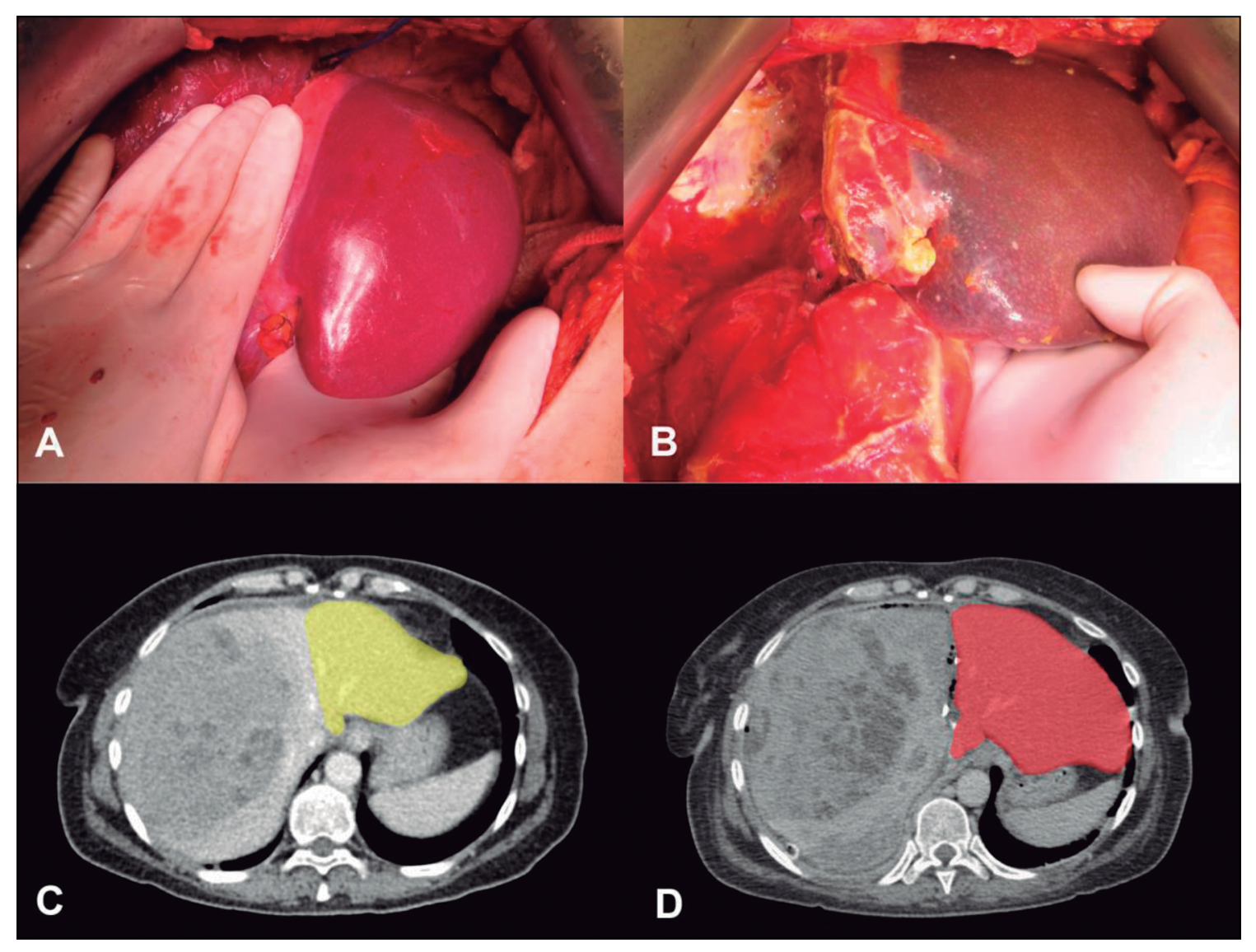

Figura 2. Fotrografía intraoperatoria parenquimatosa y TC de abdomen a nivel de la $10^{\text {a }}$ vertebra torácica que muestran la hipertrofia parenquimatosa. A: Fotografía intraoperatoria del segmento lateral remanente inicial. B: Fotografía intraoperatoria del segmento lateral remanente hipertrofiado. C: Segmento lateral inicial en amariIlo. Volumen: $285 \mathrm{cc}$ (RHF 22\%). D: Segmento lateral hipertrofiado en rojo. Volumen: $624 \mathrm{cc}$ (incremento $219 \%)$. 
hepaticoyeyuno anastomosis tutorizada que evoluciona satisfactoriamente. Finalmente, es dada de alta al $45^{\circ}$ de posoperatorio.

La paciente recibió quimioterapia adyuvante con gemcitabina y cisplatino por 6 ciclos. Al año de posoperatorio presenta recurrencia peritoneal detectada mediante PET-CT de control. La paciente se encuentra viva con 17 meses de sobrevida. El resumen se detalla en Tabla 2.

\section{Discusión}

La resección hepática constituye la única alternativa de tratamiento curativo para pacientes con patología tumoral, primaria o secundaria, del hígado ${ }^{3}$. La resecabilidad de la enfermedad hepática está determinada por la capacidad de dejar al paciente libre de enfermedad residual y con un RHF adecuado en volumen y funcionalidad para evitar desarrollar una IHP, que constituye la principal causa de mortalidad en las resecciones hepáticas ${ }^{7}$.

A inicios de la década del 90, Masatoshi Makuuchi realiza un quiebre en la evolución y el desarrollo de la $\mathrm{CH}$ al comunicar la utilidad de la EP como estrategia para aumentar el RHF en hepatectomías extendidas por colangiocarcinomas perihiliares ${ }^{8}$. Esta técnica permitió aumentar la resecabilidad de pacientes con enfermedad extensa, reduciendo la morbimortalidad asociada a IHP, y fue rápidamente adoptada por muchos grupos ${ }^{9}$ que desarrollaron estrategias de manejo de metástasis hepáticas de cáncer colorrectal en etapas, basándose en la hipertrofia del RHF, realizando LP como variante a la EP percutánea $^{10}$.

Un problema de la EP o LP para hepatectomías por etapas es que solo $2 / 3$ de los pacientes logran completar el segundo tiempo del tratamiento después de 4 a 8 semanas, ya sea por falta de hipertrofia suficiente del RHF o por progresión de la enfermedad en este periodo ${ }^{11}$. Algunos autores ${ }^{12,13}$, consideran que este periodo de observación de 4 a 8 semanas, en el cual debería realizarse quimioterapia adyuvante, es una de las ventajas de esta técnica por sobre el ALPPS, ya que representa un tiempo razonable para observar el comportamiento de la enfermedad y así evitar la realización de cirugías innecesarias en pacientes con una biología tumoral agresiva que puedan tener una progresión de la enfermedad durante este periodo.

En el año 2007, y más bien de forma fortuita, Hans Schlilt de Regensburg, Alemania tras la imposibilidad de completar una hepatectomía derecha extendida en un colangiocarcinoma hiliar (PHCC) por un RHF insuficiente y colestásico, decide no completar la cirugía y realizar una hepaticoyeyunostomía al segmento lateral transectando el parenquima a nivel del segmento IV para una correcta posición del asa y posteriormente ligó la vena porta derecha. Tras realizar un TC de control a los 8 días, se percata de la rápida regeneración del hígado remanente y decide completar la cirugía, que una semana antes, había sido imposible concretar ${ }^{5}$. Posteriormente, esta maniobra de bipartición hepática asociada a LP fue aplicada exitosamente en otros pacientes y posteriormente, adoptada por varios cirujanos principalmente alemanes, siendo el primer reporte formal el de Lang H. et al., de Mainz, Alemania con 3 casos en el Congreso E-AHPBA en Cape Down en $2011^{14}$.

Desde el año 2012, surgen las primeras series de pacientes tratados con esta técnica y los resultados han sido diversos generando una constante discusió $^{15}$. En nuestro país, el primer reporte lo realiza Uribe $\mathrm{M}$ et $\mathrm{al}^{16}$, en el año 2015 , con la descripción de un caso intervenido exitosamente en octubre de 2011. Recientemente el mismo grupo comunicó 11 casos tratados satisfactoriamente ${ }^{17}$.

Si bien, esta técnica ha demostrado tener una hipertrofia adecuada del RHF en un periodo de tiempo más corto y más constante que la EP o LP, también es cierto que la morbilidad y mortalidad asociada a la técnica fueron excesivamente elevadas al comienzo de la técnica lo que generó algunos reparos sobre su verdadera utilidad ${ }^{18,19}$.

Varios metaanálisis recientes han concluido que el ALPPS es más efectivo $(p<0,001)$ en lograr un volumen del RHF adecuado para el segundo tiempo que la LP y EP20,21.

Uno de los principales cuestionamientos a la hepatectomía en 2 tiempos con técnica de ALPPS han sido la morbilidad posoperatoria más elevada que la realizada con EP o LP. Dentro de las principales complicaciones desarrolladas en pacientes con ALPPS, la fístula biliar y las complicaciones infecciosas han liderado las estadísticas ${ }^{22}$.

Uno de los puntos más importantes para la utilización del ALPPS es su indicación, donde una adecuada selección de los casos podría disminuir la morbilidad operatoria asociada a la técnica ${ }^{23}$. El $80 \%$ de los hepatocarcinoma (HCC) $)^{24,25}$ y $10 \%$ de los colangiocarcinoma intrahepático $(\mathrm{CIH})^{26,27} \mathrm{se}$ desarrollan sobre pacientes con hepatopatía crónica o cirrosis, al igual que con EP o LP la hipertrofia parenquimatosa en estos pacientes no suele ser suficiente para evitar la IHP, así como la morbilidad entre las etapas es más elevada como en cualquier hepatectomía mayor en un paciente con cirrosis. Pese a que principalmente desde Asia existen múl- 
tiples reportes y series con resultados aceptables utilizando ALPPS en pacientes con hepatopatía crónica y cirrosis ${ }^{28-30}$, estos datos no son reproducibles en el resto del mundo y su utilización en pacientes con $\mathrm{HCC}$ y CIH debería realizarse con precaución y excepcionalmente en pacientes con hígado sano ${ }^{23}$. En nuestra serie, hemos practicado la técnica en una paciente con un gran CIH en hígado sano que requirió una trisectorectomía derecha con una ventana terapéutica muy corta. Si bien en este caso se logró una hipertrofia compensatoria adecuada del RHF y no se desarrolló una IHP, la indicación en este tipo de pacientes debe ser excepcional y con precaución.

Actualmente la técnica no está recomendada para el tratamiento de pacientes con $\mathrm{CCPH}$, patología en la cual inicialmente se desarrolló la técnica por la alta mortalidad perioperatoria en todas las series en las cuales se ha utilizado ${ }^{23}$. Esta alta mortalidad con el ALPPS se fundamenta en que el $70 \%$ de los CCPH se presentan con ictericia obstructiva ${ }^{31,32}$, lo que impide una hipertrofia compensatoria adecuada en un paciente con un RHF $<30 \%$; por tanto, esta condición, la ictericia, debería tratarse previo a realizar un manejo dirigido sobre el RHF insuficiente.

Nuestro equipo inició su experiencia en septiembre del año 2015 con el primer caso realizado frente a un hallazgo intraoperatorio discordante con el estudio imagenológico preoperatorio a nivel del segmento II. Si bien, en estos casos no hemos tenido mortalidad operatoria, los pacientes experimentaron signos de insuficiencia hepática transitoria entre el primer y segundo tiempo que remitieron con manejo médico. Probablemente, el no haber utilizado esta técnica habría significado una IHP clínicamente significativa.

La EP y la LP son alternativas menos mórbidas para mejorar el RHF insuficiente, sin embargo, la disponibilidad de EP en nuestro medio es bastante reducida en el servicio público y habitualmente su externalización termina en una excesiva demora del plan terapéutico lo cual puede hacer perder la opción de tratamiento a un paciente. En nuestro hospital contamos con EP percutánea desde el año 2017, condición que en un futuro nos permitirá evaluar globalmente todas las alternativas para el manejo de RHF insuficiente.

Los resultados con esta técnica, en términos de volumetría y funcionalidad del RHF hipertrofiado, han sido adecuados en nuestra corta experiencia, $\mathrm{y}$ todos los pacientes han tenido inicialmente una relación entre el RHF(cc)/Peso(Kg) $\leq 0,5$ que se ha sugerido como límite inferior pronóstico para el desarrollo de una IHP posterior a una hepatectomía ${ }^{13}$.

Un análisis en perspectiva, nos permite ver que, si bien se ha logrado disminuir la incidencia de IHP posterior a una gran hepatectomía, todos nuestros pacientes han recurrido de su enfermedad pese han recibido quimioterapia neoadyuvante y coadyuvante con buena respuesta inicial.

Es cierto, que se trata de pacientes con un mal pronóstico inicial, que se juegan su alternativa de curación en un cirugía compleja como la hepatectomía en 2 tiempos clásica o con la técnica de ALPPS, que en nuestra corta experiencia esta técnica ha permitido una oportunidad más rápida de resección completa de todas las lesiones hepáticas que la variante convencional en 2 tiempos; pero también se debe tener en consideración que al igual que nuestro grupo, actualmente, los seguimientos oncológicos con más de 24 meses utilizando esta técnica son escasos con medias de libre de enfermedad de 12 meses $^{33}$ y sobrevidas globales a 1 año sobre el $80 \%{ }^{19,33,34}$, principalmente en metástasis colorrectales.

Recientemente los resultados iniciales del único estudio prospectivo aleatorizado comparando ALPPS versus hepatectomía convencional en etapas para pacientes con metastasis hepáticas de cáncer colorectal (LIGRO trial) ${ }^{35}$ evaluó como objetivo primario la tasa de resecabiliad y como secundario la tasa de complicaciones, radicalidad y mortalidad a 90 días. ALPPS resultó ser más efectivo que la hepatectomía convencional por etapas en tasa de resecabilidad con comparables tasas de complicaciones y mortalidad a 90 días. En este estudio no se han evaluado resultados oncológicos a largo plazo, con seguridad, en los próximos años contaremos con resultados que proporcionen la evidencia necesaria para tomar mejores decisiones terapéuticas en nuestros pacientes.

\section{Conclusión}

En nuestra experiencia inicial, la técnica de ALPPS logró aumentar el RHF permitiendo resecar pacientes que inicialmente tenían un RHF insuficiente para ser sometidos a una hepatectomía mayor.

\section{Responsabilidades éticas}

Protección de personas y animales. Los autores declaran que para esta investigación no se han realizado experimentos en seres humanos ni en animales.

Confidencialidad de los datos. Los autores declaran que en este artículo no aparecen datos de pacientes.

Derecho a la privacidad y consentimiento informado. Los autores declaran que en este artículo no aparecen datos de pacientes. 


\section{Referencias}

1. He VJ. Professor Henri Bismuth: the past, present and future of hepatobiliary surgery. Hepatobiliary Surg Nutr. 2013;2:236-8.

2. Page AJ, Kooby DA. Perioperative management of hepatic resection. J Gastrointest Oncol J Gastrointest Oncol. 2012;33:19-27.

3. Fan MH, Chang AE. Resection of liver tumors: Technical aspects. Surg Oncol. 2002;10:139-52.

4. Liau KH, Blumgart LH, DeMatteo RP. Segment-oriented approach to liver resection. Surg Clin North Am. 2004;84:543-61.

5. De Santibañes E, Clavien PA. Playing play-doh to prevent postoperative liver failure: The "aLPPS" approach. Ann Surg. 2012;255:415-7.

6. Lim MC, Tan CH, Cai J, Zheng J, Kow AWC. CT volumetry of the liver: Where does it stand in clinical practice? Clin Radiol. 2014;69:887-95.

7. Rahbari NN, Garden OJ, Padbury R, Brooke-smith M. Posthepatectomy liver failure: A definition and grading by the International Study Group of Liver Surgery (ISGLS). Surgery. 2011;149:71324.

8. Makuuchi M, Thai B, Takayasu K, Takayama T, Kosuge T, Gunven P, et al. Preoperative portal embolization to increase safety of major hepatectomy for hilar bile duct carcinoma: a preliminary report. Surgery. 1990;107:521-7.

9. Bismuth H, Adam R, Lévi F, Farabos C, Waechter F, Castaing D, et al. Resection of Nonresectable Liver Metastases from Colorectal Cancer After Neoadjuvant Chemotherapy. Ann Surg. 1996;224:50922.

10. Adam R, Laurent A, Azoulay D, Castaing D, Bismuth H. Two-stage hepatectomy: A planned strategy to treat irresectable liver tumors. Ann Surg. 2000;232:777-85.

11. Schadde E, Ardiles V, Slankamenac K, Tschuor C, Sergeant G, Amacker N, et al. ALPPS offers a better chance of complete resection in patients with primarily unresectable liver tumors compared with conventional-staged hepatectomies: Results of a multicenter analysis. World J Surg. 2014;38:1510-19.

12. Khan AS, García-Aroz S, Ansari MA
Atiq SM, Senter-Zapata M, Fowler K, et al. Assessment and optimization of liver volume before major hepatic resection: Current guidelines and a narrative review. Int J Surg. 2018;52:74-81.

13. Shindoh J, Vauthey JN, Zimmitti G, Curley SA, Huang SY, Mahvash A, et al. Analysis of the efficacy of portal vein embolization for patients with extensive liver malignancy and very low future liver remnant volume, including a comparison with the associating liver partition with portal vein ligation for staged hepatectomy approa. J Am Coll Surg. 2013;217:126-33.

14. Baumgart J, Lang S, Lang H. A new method for induction of liver hypertrophy prior to right trisectionectomy: a report of three cases. HPB (Oxford). 2011;13:71-2.

15. Baumgart J, Farkas SA, Fichtner-feigl S, Lorf T. Right Portal Vein Ligation Combined With In Situ Splitting Induces Rapid Left Lateral Liver Lobe Hypertrophy Enabling 2-Staged Extended Right Hepatic Resection in Small-for-Size Settings. Ann Surg. 2012;255:24-6.

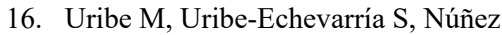
J, Martínez W, Paqui L, Camacho $\mathrm{J}$, et al. Asociación de la partición hepática y ligadura de la vena porta para hepatectomía por etapas, un nuevo procedimiento en cirugía hepática. Rev Chil Cir. 2015;67:185-90.

17. Uribe M, Uribe-Echevarría S, Mandiola C, Zapata MI, Riquelme F, Romanque P. Insight on ALPPS - Associating Liver Partition and Portal Vein Ligation for Staged Hepatectomy - mechanisms: activation of mTOR pathway. HPB (Oxford). 2018;1-10.

18. Lau WY, Lai EC, Lau SH. Associating liver partition and portal vein ligation for staged hepatectomy: the current role and development. Hepatobiliary Pancreat Dis Int. 2017;16:17-26.

19. Olthof PB, Coelen RJS, Wiggers JK, Koerkamp BG, Malago M, Hernándezalejandro R, et al. High mortality after ALPPS for perihilar cholangiocarcinoma: case-control analysis including the fi rst series from the international ALPPS registry. HPB (Oxford). 2017;19:381-7.

20. Eshmuminov D, Raptis DA, Linecker M, Wirsching A, Lesurtel M, Clavien P.
Meta-analysis of associating liver partition with portal vein ligation and portal vein occlusion for two-stage hepatectomy. Br J Surg. 2016;103:1768-82.

21. Huang H, Liu W, Wang A, Bian J, Wang $\mathrm{S}, \mathrm{Wu} \mathrm{L}$, et al. Comparison of portal vein embolization, portal vein ligation, associating liver partition and portal vein ligation for staged hepatectomy in cases with a small future liver remnant: a network meta-analysis. Transl Cancer Res. 2017;6:826-33.

22. Truant $\mathrm{S}$, Scatton $\mathrm{O}$, Dokmak $\mathrm{S}$, Regimbeau JM, Lucidi V, Laurent A, et al. Associating liver partition and portal vein ligation for staged hepatectomy (ALPPS): Impact of the inter-stages course on morbi-mortality and implications for management. Eur J Surg Oncol. 2015;41:674-82.

23. Oldhafer KJ, Stavrou GA, van Gulik TM. ALPPS-Where Do We Stand, Where Do We Go? Ann Surg. 2016;263:839-41.

24. Chalasani N, Marrero J a, Ahn J, Rajender Reddy K. ACG Clinical Guideline: The Diagnosis and Management of Focal Liver Lesions. Am J Gastroenterol. 2014;109:1328-47.

25. Guidelines CP. EASL-EORTC Clinical Practice Guidelines: Management of hepatocellular carcinoma. Eur J Cancer. 2012;48:599-641.

26. Dodson RM, Weiss MJ, Cosgrove D, Herman JM, Kamel I, Anders R, et al. Intrahepatic cholangiocarcinoma: Management options and emerging therapies. J Am Coll Surg. 2013;217:73650.

27. Bridgewater J, Galle PR, Khan SA, Llovet JM, Park J, Patel T, et al. Guidelines Guidelines for the diagnosis and management of intrahepatic cholangiocarcinoma. J Hepatol. 2014;60:1268-89.

28. Cai X, Tong $\mathrm{Y}$, Yu H, Liang X, Wang Y, Liang Y, et al. The ALPPS in the Treatment of Hepatitis B - Related Hepatocellular Carcinoma With Cirrhosis: A Single-Center Study and Literature Review. Surg Innov. 2017;24:358-64.

29. Chan ACY, Feshk M, Fres F, Chok $\mathrm{K}$, Ms M, Fres F, et al. Impact of split completeness on future liver remnant hypertrophy in associating liver partition and portal vein ligation for staged 
hepatectomy (ALPPS) in hepatocellular carcinoma: Complete-ALPPS versus Surgery. 2016;161:357-64.

30. Xiao L, Zheng JLS. Totally laparoscopic ALPPS in the treatment of cirrhotic hepatocellular carcinoma. Surg Endosc. 2015;9:2800-1.

31. Yokoyama Y, Nagino M, Nimura Y. Mechanism of impaired hepatic regeneration in cholestatic liver. Journal of Hepato-Biliary-Pancreatic Surgery. 2007:14;159-66.

32. Wiggers JK, Koerkamp BG, Cieslak KP, Doussot A, van Klaveren D, Allen $\mathrm{PJ}$, et al. Postoperative Mortality after Liver Resection for Perihilar Cholangiocarcinoma: Development of a Risk Score and Importance of Biliary Drainage of the Future Liver Remnant. J Am Coll Surg. 2016;223:321-31.

33. Wanis K, Ardiles V, Álvarez F, Tunabraham ME, Linehan D, Santibañes $\mathrm{E}$, et al. Intermediate-term survival and quality of life outcomes in patients with advanced colorectal liver metastases undergoing associating liver partition and portal vein ligation for staged hepatectomy. Surgery. 2017;1-7.

34. Hasan I Al, Tun-Abraham ME, Wanis K, García-Ochoa C, Levstik MA,
Al-Judaibi B, et al. Optimizing associated liver partition and portal vein ligation for staged hepatectomy outcomes: Surgical experience or appropriate patient selection? J Can Chir. 2017;60:408-15.

35. Sandström P, Røsok B, Sparrelid E, Larsen P, Larsson A, Lindell G, et al. ALPPS Improves Resectability Compared With Conventional Two-stage Hepatectomy in Patients With Advanced Colorectal Liver Metastasis: Results From a Scandinavian Multicenter Randomized Controlled Trial (LIGRO Trial). Ann Surg. 2018;267:833-40. 